\title{
Designing of Standard-Size Shapes of Heads for Selection from Customized and Mass-Produced Headwear
}

\author{
Konstantin KARAVAEV, Maxim TRUPHANOV \\ Elasizer Srl., Milan, Italy
}

DOI: $10.15221 / 18.120$ http://dx.doi.org/10.15221/18.120

\begin{abstract}
The presented research introduces the method along with soft and hardware which allows to design gear and headwear and provides further easy selection of the necessary items among the standard mass produced goods which best match the individual requirements of a client.

The paper introduces the results of the research and the developed method along with the description of special soft and hardware which allows the fast and precise selection of an individual item of gear or headwear using the commonly available means.

The advantage of the presented method and the means is the combined use of information and data on available size ranges of headwear and protective sports headgear together with individual size and shape of a client's head, which guarantees reasonable price/quality ratio and allows a client to choose that precise item among the mass-produced goods which provides maximum comfort. Another distinctive feature of the presented approach is that it allows fast selection of the required item by means of creation and further use of the unique code index which defines the shape, size and other individual characteristics of a particular client.

To sum up, the devoted solutions which are introduced in this presentation combine the advantages of the customized approach and the selection from the mass-produced gear thus providing the fast and accurate way to choose a particular sports gear item or headwear that best matches the individual requirements of a client.
\end{abstract}

Keywords: headwear, helmet, measurement, customized, mass-produced, selection

Modern technology features two completely different approaches to selection of protective gear (for instance, helmets for various sports) and headwear. One of these approached is based totally on individual precise measurement of required sizes of a customer's head with further production of an item of gear or headwear. This particular approach is costly and time-consuming, hence often not suitable for most customers, because the desired item is expensive and it takes a lot of time to produce it. The other approach only allows to choose a ready-made item among the mass produced items, which as a rule provides comfort only for a limited number of customers due to the low accuracy of matching of an item to a customer's head.

The presented research introduces the method along with soft and hardware which allows to design gear and headwear and provides further easy selection of the necessary items among the standard mass produced goods which best match the individual requirements of a customer.

One of the key tasks in the field of virtual selection of clothing, protective equipment, sports equipment is the creation of an effective technical solution that is accessible to the mass consumer and allows anyone to choose the items best matching the individual anthropometric characteristics among the mass-produced goods (clothing, footwear, protective gear). Moreover, the conducted analysis showed that if just standard characteristics are used (such as the size of width) it provides the customer only with approximate information about convenience and safety of a product (especially if it is a protective gear item such as a helmet).

Along with this, there are known solutions in the luxury segment of the clothing market that allow to perform individual measurements by means of a stationary relatively expensive scanner in stationary conditions of a store and develop a virtual model of clothing or footwear for a customer and order it from a particular manufacturer. Obviously, this variant deals with a lot of discomfort for a customer, because a customer must personally come to the store where the scanner is installed, spend his/her time, and wait for the product to be manufactured, all that takes much time and is rather inconvenient. 
Thus, current technical solutions allow either to produce a truly individual piece of clothing but are inconvenient for a customer or to buy a ready-made item which may or may not provide the desired comfort and safety, which causes certain risks if it is an item of protective equipment.

When comparing the item of equipment and a customer's body part, a specially developed code is used that determines the individual anthropometric features and subjective preferences of a customer. The principle of measuring and constructing the subsequent standardized code, which determines the anthropometric characteristics of the product in the encoded form is as follows.

As the survey of the number of respondents shows, the most common individual subjective and objective characteristics and preferences of people who use the sports protective goods were determined. The results of the survey were grouped according to experience, age, lifestyle and other characteristics of the respondents. Further, the statistical analysis was conducted, which allowed to select the most significant sets of characteristics and determine the vector of the parameters of the target equipment (for example, motorcycle helmets), which allows in a compact form to describe the requirements for the goods and choose a particular product among the available ones. Thus, the developed approach at this stage allows to provide the fast and accurate selection of clothing, footwear or protective sports equipment by forming a compact, standardized and at the same time unique description of individual characteristics of a customer. This can be used as a short code that determines the basic anthropometric features, as well as an extended code that takes into account the complete objective description and individual subjective features of a customer.

The measurement can be performed either by an individual communication and measurement device or by a precision scanner.

The developed system represents the cloud storage of data and programs with the information processing algorithms, a mobile phone or a tablet, and a stationary scanner which is used for individual cases.

The beginning of the scanning process goes as follows: from the cloud communication module the individual communication and measurement device or precision scanner reads the necessary programs and algorithms for scanning, then the scanned part of the body is positioned in a specified manner relative to the sensors of the precision scanner or the optical sensor of the individual communication and measurement device. Further, the precision scanner or the individual communication and measurement device registers images of the surface of a part of the body in specified positions, determines the key features, and transfers the generated data sets to the cloud storage. The cloud storage according to the stored algorithms and programs calculates the parameters of the shape of the customer's body part and builds a unique vector which describes the customer's anthropometric features.

Next, the process of selection of the most optimal (closest to the given criteria) item of equipment for this particular customer among the available data on the target product requested by the customer (for example, a helmet) is performed.

The resulting parameter vector of a customer allows the following:

- on the basis of calculation choose the best matching item of equipment taking into account the price of the goods;

- receive the alphanumeric code that determines the size, shape and other significant parameters of a particular item of clothing, which provides an opportunity for further impersonal selection of goods best matching a particular customer using this code.

- calculation of the factor of conformity with the figure or part of a customer's body, which provides a preliminary assessment of comfort and individual fit of the selected clothing.

In this case, the factor of the product's conformity to the figure or a part of a customer's body is an indication of the degree of closeness of the figure or a part of the customer's body to the figure or a part of the body of a virtual consumer for which a particular standard product was manufactured. When searching for a ready-made product, the factor of the product's conformity to the figure of a customer or a part of a customer's body is used.

Thus, as a result of the operations described above, a customer receives the list of goods which best match his/her figure or a body part. 
The presented approach evolved from the previously developed approaches is based on the special stationary device for scanning the surface of a customer's head which includes a mass produced camera or a smartphone. The presented approach is the innovative technology for the selection of individual headwear and clothing.

The advantage of the presented method and the means is the combined use of information and data on available size ranges of headwear and protective sports headgear together with individual size and shape of a customer's head, which guarantees reasonable price/quality ratio and allows a customer to choose that precise item among the mass-produced goods which provides maximum comfort.

Another distinctive feature of the presented approach is that it allows fast selection of the required item by means of creation and further use of the unique code index which defines the shape, size and other individual characteristics of a particular customer.

To sum up, the devoted solutions which are introduced in this presentation combine the advantages of the customized approach and the selection from the mass-produced gear thus providing the fast and accurate way to choose a particular sports gear item or headwear that best matches the individual requirements of a customer.

The research shows that when using a mobile phone the current calculating error for linear values when scanning individual parts of the body is less than $1 \%$. When using a stationary scanner, the error of less than $0.7 \%$ hence the accuracy can potentially be increased approximately twice due to the implementation of new algorithms not mentioned in this paper.

Thus, the introduced technical solution allows to create a compact, coded, accurate description of a customer's anthropometric characteristics in accordance with the selected item of clothing, footwear or protective equipment. The introduced solution allows a customer to perform an accurate measurement of his/her body or body part and to choose in a short time the best matching item to him of clothing or equipment without complicated technical means. 